\title{
A NEW COMPUTATIONAL APPROACH TO NUCLEAR AEROSOL PROBLEMS
}

\author{
KASEM N. ABOTEL and EDWARD W. LARSEN \\ Department Of Nuclear Engineering \\ University of Michigan \\ Ann Arbor, Michigan 48109, USA
}

(Received 2nd May 1990; in revised form 18 July 1990)

\begin{abstract}
The nonlinear kinetic aerosol equation, describing the time evolution of an aerosol distribution within a well-stirred container, is formulated in a mathematically "conservative" form. A numerical method is then developed for which conservation of mass is automatically satisfied. This procedure simplifies the derivation of conservative numerical schemes by reducing the number of approximations that must be made. Comparisons between an exact solution of the kinetic aerosol equation and numerical approximations show the following: numerical solutions based on the conservative form of the kinetic equation are more accurate and are obtained more efficiently than numerical solutions based on the standard "nonconservative" form of the kinetic equation.
\end{abstract}

\section{INTRODUCTION}

In recent years, much effort has been spent on numerically predicting the release and transport of nuclear aerosols following a postulated nuclear accident, in order to obtain a better understanding of the consequences of such accidents. The aerosol problem contains many physical processes. In this work, we concentrate on one aspect of this problem: the computer modeling of the coagulation of aerosols within a well-stirred container.

Several computer codes have been written to solve this problem (Silberberg (1979), Abbey (1985)). These codes, which use numerical methods based on a standard nonconservative form of the kinetic aerosol equation, have several disadvantages. First, they can be costly because the volume of the aerosol particles may span over ten orders of magnitude, and for the numerical methods used in these codes, a grid containing very many points is often required. Second, to obtain numerical schemes that conserve mass, more approximations must be made than numerical schemes for other problems usually require. [As we will see later, in obtaining the discretization used in the Pardiseko code (Dunbar (1984), Bunz (1984)), one must approximate certain integrals that are very difficult to evaluate.]

In this paper we formulate the kinetic aerosol equation, describing the time evolution of the aerosol density, in a mathematically "conservative" form. In this form, the nonlinear terms that describe the coagulation process can be shown to conserve mass by a trivial mathematical procedure. In the conventional nonconservative form, the coagulation terms also conserve mass, but showing this requires a substantial amount of algebra. An advantage of the conservative form is that it permits a very simple derivation of accurate numerical schemes that conserve mass. To obtain mass-conserving numerical schemes for the "nonconservative" form of the aerosol equation, one must employ extra approximations that are based on physical, rather than mathematical, principles. In this paper we show that numerical methods based on the conservative form of the aerosol equation are generally 
more accurate and are obtained more efficiently than methods based on the nonconservative form of the equation.

In Sec. 2 we review the kinetic aerosol equation, written in the conventional nonconservative form. In Sec. 3 we review the derivation of a well-known mass-conserving numerical method for this equation. In Sec. 4 we derive the conservative form of the kinetic aerosol equation, and we develop a numerical method for this equation. In Sec. 5 we examine the positivity of our method. In Sec. 6 we discuss numerical results obtained using a Runge-Kutta time-discretization method, together with the two methods developed in Secs. 3 and 4 of this paper. We conclude with a brief discussion in Sec. 7.

\section{THE KINETIC AEROSOL EQUATION FOR A WELL-STIRRED CONTAINER}

The quantity of interest in aerosol problems is $n\left(r, v_{2} t\right) d^{3} r d v$, the expected number of aerosol particles in an incremental volume of space $d^{3} r$ about the point $r$, having volumes in the incremental range $v$ to $v+d v$, at time $t$. These particles are subject to the effects of atmospheric motion, Brownian motion, and gravity. When two particles collide, they may coagulate to form a single new particle whose mass equals the sum of the masses of the original particles. As this process continues and particles become more massive, gravity becomes dominant and particles fall out of the suspension.

Within a container in which the aerosol is well-stirred, the aerosol density $n$ is independent of the position variable, and the kinetic aerosol equation describing the time evolution of $n$ can be written as (Silberberg (1979), Abbey (1985))

$$
\begin{gathered}
\frac{\partial}{\partial t} n(v, t)=\frac{1}{2} \int_{0}^{v} d u n(u, t) \phi(u, v-u) n(v-u, t)-n(v, t) \int_{0}^{\infty} d u \phi(u, v) n(u, t) \\
-R(v, t) n(v, t)+S(v, t),
\end{gathered}
$$

with the initial condition

$$
n(v, 0)=n_{0}(v)
$$

The various terms in Eq. (1) are defined by:

$\phi(u, v) n(u, t) n(v, t) d u d v=$ the rate at which aerosol particles in $d u$ about $u$ and in $d v$ about $v$ coagulate. ( $\phi$ is the coagulation kernel.)

$R(v, t) n(v, t) d v=$ the rate at which aerosol particles in $d v$ about $v$ are removed, due to gravitational settling to the floor, diffusion to the walls, and leakage. ( $R$ is the removal rate.)

$S(v, t) d v=$ the rate at which aerosol particles are produced in $d v$ about $v$ due to sources. ( $S$ is the source rate.)

The first integral in Eq. (1) represents the rate at which particles with volume $v$ are produced as a result of collision between particles of volume $u$ and $v-u$. (The factor $1 / 2$ occurs because in integrating $u$ from 0 to $v$, particles with volumes $u$ and $v-u$ are counted twice.) The second integral represents the rate of loss of particles with volume $v$, as a result of collisions with other particles. One can show that the nonlinear terms in Eq. (1), describing the coagulation process, conserve mass: 


$$
\int_{0}^{\infty} d v v\left[\frac{1}{2} \int_{0}^{v} d u n(u, t) \phi(u, v-u) n(v-u, t)-n(v, t) \int_{0}^{\infty} d u \phi(u, v) n(u, t)\right]=0 \text {. }
$$

Therefore, if one multiplies Eq. (1) by $v$ and integrates over all $v$, one obtains the following balance equation:

$$
\frac{d}{d t} \int_{0}^{\infty} v n(v, t) d v=-\int_{0}^{\infty} v R(v, t) n(v, t) d v+\int_{0}^{\infty} v S(v, t) d v
$$

This states that the rate of change of the total particle volume (or mass) equals the rate at which mass is produced by sources minus the rate at which it is removed. The coagulation process neither adds nor subtracts mass from the system.

The identity $(3 a)$ can be established by routine but lengthy manipulations. Because this identity does not immediately follow using elementary operations, we refer to Eq. (1) as a nonconservative form of the kinetic aerosol equation. Special attention must be devoted to any numerical method based on Eq. (1) in order to make it conserve mass - that is, in order to make it satisfy a discrete version of $\mathrm{Eq}$. (3a). In the next section, we consider such a method.

\section{FINITE DIFFERENCE METHOD (PARDISEKO)}

In this section we summarize the numerical method employed in the Pardiseko code (Dunbar (1984), Bunz (1984)). To begin, let us divide the volume range into $I$ cells whose widths vary logarithmically. That is, the edges $v_{i+1 / 2}$ of these cells are defined by

$$
v_{i+1 / 2}=v_{\min }\left(\frac{v_{\max }}{v_{\min }}\right)^{i / I} \quad, \quad 0 \leq i \leq I
$$

where $v_{1 / 2}=v_{\min }$ and $v_{I+1 / 2}=v_{\max }$ are the prescribed minimum and maximum volumes and the width $h_{i}$ of the $i-t h$ cell is defined by

$$
h_{i}=v_{i+1 / 2}-v_{i-1 / 2}
$$

(It is not necessary to use this particular logarithmic grid, but it is convenient to do so, and we shall use it throughout this paper.) We also make the approximations

$$
\begin{gathered}
\mathrm{n}_{\mathrm{i}}(\mathrm{t}) \approx n(v, t), v_{i-1 / 2}<v<v_{i+1 / 2}, \\
\mathrm{R}_{\mathrm{i}}(\mathrm{t}) \approx R(v, t), v_{i-1 / 2}<v<v_{i+1 / 2}, \\
\mathrm{~S}_{\mathrm{i}}(\mathrm{t}) \approx S(v, t), v_{i-1 / 2}<v<v_{i+1 / 2}, \\
\phi_{j i}=\phi(u, v), u_{j-1 / 2}<u<u_{j+1 / 2}, v_{i-1 / 2}<v<v_{i+1 / 2},
\end{gathered}
$$

and we define 


$$
\theta_{i}(v)=\left\{\begin{array}{cc}
1 & v_{i-1 / 2}<v<v_{i+1 / 2} \\
0 & \text { otherwise }
\end{array}\right.
$$

For a general function $G$, one can prove the following identity (Dunbar (1984)):

$$
\int_{v_{i-1 / 2}}^{v_{i+1 / 2}} d v \int_{0}^{v} d u G(u, v-u)=\int_{v_{1 / 2}}^{v_{i+1 / 2}} d w \int_{0}^{v_{i+1 / 2}} d u G(u, w) \theta_{i}(u+w)
$$

Integrating Eq. (1) over $v_{i-1 / 2}<v<v_{i+1 / 2}$ and using the identity (5b), we obtain

$$
\begin{aligned}
& \frac{d}{d t} n_{i}(t) h_{i} \approx \frac{1}{2} \int_{v_{1 / 2}}^{v_{i+1 / 2}} d w \int_{v_{1 / 2}}^{v_{i+1 / 2}} d u n(u, t) \phi(u, w) n(w, t) \theta_{i}(u+w) \\
& -\int_{v_{i-1 / 2}}^{v_{i+1 / 2}} d v n(v, t) \int_{v_{1 / 2}}^{v_{I+1 / 2}} d u \phi(u, v) n(u, t)-R_{i}(t) n_{i}(t) h_{i}+S_{i}(t) h_{i} \\
& =\sum_{j=1}^{i} \sum_{k=1}^{i} \frac{1}{2} \int_{v_{j-1 / 2}}^{v_{j+1 / 2}} d w \int_{v_{k-1 / 2}}^{v_{k+1 / 2}} d u n(u, t) \phi(u, w) n(w, t) \theta_{i}(u+w) \\
& -\int_{v_{i-1 / 2}}^{v_{i+1 / 2}} d v n(v, t) \sum_{k=1}^{I} \int_{v_{k-1 / 2}}^{v_{k+1 / 2}} d u \phi\left(u, v_{i}\right) n(u, t)-R_{i}(t) n_{i}(t) h_{i}+S_{i}(t) h_{i} \\
& \approx \frac{1}{2} \sum_{j=1}^{i} \sum_{k=1}^{i} n_{j}(t) n_{k}(t) \phi_{k j}\left\{\int_{v_{j-1 / 2}}^{v_{j+1 / 2}} d w \int_{v_{k-1 / 2}}^{v_{k+1 / 2}} d u \theta_{i}(u+w)\right\} \\
& -n_{i}(t) h_{i} \sum_{k=1}^{I} n_{k}(t) \phi_{k i} h_{k}-R_{i}(t) n_{i}(t) h_{i}+S_{i}(t) h_{i}
\end{aligned}
$$

Thus, if we define

$$
\gamma_{i j k}=\frac{1}{h_{j} h_{k}} \int_{v_{j-1 / 2}}^{v_{j+1 / 2}} d w \int_{v_{k-1 / 2}}^{v_{k+1 / 2}} d u \theta_{i}(u+w)
$$

then we obtain

$$
\begin{aligned}
\frac{d}{d t} n_{i}(t) h_{i}= & \frac{1}{2} \sum_{j=1}^{I} \sum_{k=1}^{I} n_{j}(t) n_{k}(t) \gamma_{i j k} \phi_{j k} h_{j} h_{k}-n_{i}(t) h_{i} \sum_{j=1}^{I} \phi_{j i} n_{j}(t) h_{j} \\
& -R_{i}(t) n_{i}(t) h_{i}+S_{i}(t) h_{i} \quad, \quad 1 \leq i \leq I
\end{aligned}
$$


This is a coupled system of $I$ ordinary differential equations having two main deficiencies: the constants $\gamma_{i j k}$, defined by Eq. (7), are difficult to evaluate, and the nonlinear coagulation terms in Eq. (8) do not generally conserve mass. To circumvent both these difficulties, one simply replaces the definition (7) of $\gamma_{i j k}$ by an easily-computed and physically-motivated definition. To do this, let us suppose that every particle in the $j$-th cell has volume $v_{j}$ - the mean volume in this cell. Also, let us suppose that particles with volumes $v_{j}$ and $v_{k}$ coagulate to form a single new particle with volume $v_{j}+v_{k}$. In general, this new volume is not a grid point. Therefore, we determine a grid point $v_{i}$ such that

$$
v_{i}<v_{j}+v_{k}<v_{i+1} \quad(\text { if } i<I)
$$

and we conceptually create two particles, one with mass $v_{i}$, the other with mass $v_{i+1}$, whose weights sum to one (to preserve the number of new particles) and whose volumes sum to $v_{j}+v_{k}$ (to preserve mass). Interpreting $\gamma_{i, j, k}$ as the contribution to particles of mass $v_{i}$ when two smaller particles of mass $v_{j}$ and $\nu_{k}$ coagulate, we let $\gamma_{i, j, k}$ and $\gamma_{i+1, j, k}$ be the appropriate weights. Then:

$$
\begin{gathered}
\gamma_{i, j, k}+\gamma_{i+1, j, k}=1 \\
\gamma_{i, j, k} v_{i}+\gamma_{i+1, j, k} v_{i+1}=v_{j}+v_{k}
\end{gathered}
$$

Solving these equations we obtain, for $j, k$, and $i$ satisfying Eq. (9a),

$$
\gamma_{m, j, k}=\left\{\begin{array}{cc}
\frac{v_{i+1}-\left(v_{j}+v_{k}\right)}{v_{i+1}-v_{i}} & m=i, \\
\frac{\left(v_{j}+v_{k}\right)-v_{i}}{v_{i+1}-v_{i}} & m=i+1, \\
0 & \text { otherwise. }
\end{array}\right.
$$

If there is no grid point $v_{i}$ such that Eq. $(9 a)$ is satisfied, i.e., if

$$
v_{j}+v_{k}>v_{I}
$$

where $v_{l}$ is the largest volume in the grid, then we create a particle with mass $v_{l}$, with an appropriate weight so that mass is preserved. For $j$ and $k$ satisfying Eq. $(10 a)$, we obtain

$$
\gamma_{i, j, k}=\left\{\begin{array}{lc}
\frac{v_{j}+v_{k}}{v_{I}} & i=I, \\
0 & 1 \leq i \leq I-1 .
\end{array}\right.
$$

The numerical method used in the Pardiseko code is given by Eqs. (8) - (10). In the remainder of this paper, we shall describe this as the "Nv" method - the "N" referring to the fact that the method is based on a nonconservative form of the aerosol equation, and the " $v$ " referring to the fact that the volume variable is used. One can show that this method conserves mass, in the sense that the following discrete version of Eq. ( $3 a$ ) holds: 


$$
\sum_{i=1}^{l} v_{i}\left[\sum_{j=1}^{I} \sum_{k=1}^{I} n_{j}(t) n_{k}(t) \gamma_{i j k} \phi_{j k} h_{j} h_{k}-n_{i}(t) \sum_{j=1}^{I} \phi_{j i} n_{j}(t) h_{j}\right] h_{i}=0
$$

However, a difficulty now occurs which is due to the finiteness of the grid: in the absence of removal terms, as time increases and the aerosol particles become heavier, all of the particles will eventually settle into the $I-t h$ cell. This situation is unphysical because in a real problem, when particles attain a sufficiently large size, gravitational effects remove them. For this reason, the "simplest" aerosol problem - one with no removal and no sources - is a very difficult one to treat numerically for large times. To do this operationally, one would solve such a problem with a given grid, but then at some point in time, when the number of particles in the $I-t h$ cell is large enough, one would have to declare that the solution for larger times is incorrect. If the calculation had begun with a grid with the same resolution but extending over a broader range of volumes, then the time beyond which the solution is unphysical would be larger. These difficulties must occur with any mass-conserving scheme on a finite grid in the absence of removal terms.

We have shown that to obtain a practical mass-conserving method, one must employ a physical approximation to evaluate the constants $\gamma_{i, j, k}$. In part, this occurs because Eq. (1) does not, by means of simple operations that can be reproduced by discretized versions of Eq. (1), conserve mass. Our contention is that if Eq. (1) is rewritten in such a way that simple operations that can be reproduced by numerical schemes lead to mass conservation, then numerical schemes based on this equation need not contain any unnecessary physical approximations. This is the subject of the rest of this paper.

\section{THE CONSERVATIVE FORM OF THE KINETIC AEROSOL EQUATION}

By straightforward manipulations, Eq. (1) may be written as:

$$
\frac{\partial}{\partial t} v n(v, t)=-\frac{\partial}{\partial v} F(v, t)-v R(v, t) n(v, t)+v S(v, t)
$$

where

$$
F(v, t)=\int_{0}^{v} d u \int_{\nu-u}^{\infty} d w u n(u) \phi(u, w) n(w)
$$

Physically, $F(v, t)$ is the rate at which mass is transferred from particles with volumes less than $v$ to particles with volumes greater than $v$. We term $F$ the mass agglomeration density; $F$ plays a role similar to that of $q$, the slowing down density, in neutron thermalization problems (Williams (1966)). It is obvious, both from the definition (12b) and the physical interpretation of $F$, that $F(0, t)=0$. Also, under the assumption that particles are removed by gravitational effects when they become sufficiently massive, we have $n(\infty, t)=0$, so $F(\infty, t)=0$. It immediately follows that, integrating Eq. $(12 a)$ over all $v$, the balance equation $(3 b)$ is obtained. Eq. ( $3 b)$ follows from both Eq. (1) and Eqs. (12), but the algebra necessary to show that the coagulation terms cancel out of Eqs. (1) is nontrivial, whereas the algebra needed to show that the coagulation terms cancel out of Eq. (12) is trivial. For this reason, we refer to Eqs. (12) as the conservative form of the kinetic aerosol equation.

We now derive a simple mass-conserving numerical scheme for Eqs. (12). Integrating Eq. (12a) over $v_{i-1 / 2}<v<v_{i+1 / 2}$, we obtain: 


$$
\frac{d}{d t} v_{i} n_{i}(t) h_{i}=F\left(v_{i-1 / 2}, t\right)-F\left(v_{i+1 / 2}, t\right)-v_{i} R_{i}(t) n_{i}(t) h_{i}+v_{i} S_{i}(t) h_{i}
$$

It remains to approximate $F$. However, we first observe that no matter how $F$ is approximated, as long as we require

$$
\begin{gathered}
F\left(v_{1 / 2}, t\right)=0, \\
F\left(v_{I+1 / 2}, t\right)=0,
\end{gathered}
$$

then it follows from Eq. (13) that

$$
\frac{d}{d t} \sum_{i=1}^{I} v_{i} n_{i}(t) h_{i}=-\sum_{i=1}^{I} v_{i} R_{i}(t) n_{i}(t) h_{i}+\sum_{i=1}^{I} v_{i} S_{i}(t) h_{i} .
$$

This is just a discretized form of the balance equation $(3 b)$, stating that the discretized coagulation operator neither creates nor destroys mass. Therefore, no matter how $F\left(v_{i+1 / 2}, t\right)$ is defined for $1 \leq i \leq I-1$, the resulting numerical scheme will conserve mass. To evaluate $F\left(v_{i+1 / 2}, t\right)$ for $1 \leq i \leq I-1$, we take

$$
\begin{gathered}
F\left(v_{i+1 / 2}, t\right)=\int_{v_{1 / 2}}^{v_{i+1 / 2}} d u \int_{v_{i+1 / 2}-u}^{v_{t+1 / 2}} d w u n(u, t) \phi(u, w) n(w, t) \\
\approx \sum_{j=1}^{i} v_{j} n_{j}(t) h_{j}\left\{\int_{j=1}^{v_{j+1 / 2}^{v_{j+1 / 2}} d u u n(u, t) \int_{v_{j+1 / 2}}^{v_{i+1 / 2}-v_{j}} d w \phi\left(v_{j}, w\right) n(w, t)} \int_{v_{i+1 / 2}-v_{j}}^{\int_{v_{j}}} d w\left(v_{j}, w\right) n(w, t)+\sum_{m=k+1}^{I} \int_{v_{m-1 / 2}}^{v_{m+1 / 2}} d w \phi\left(v_{j}, w\right) n(w, t)\right\} \\
\approx \sum_{j=1}^{i} v_{j} n_{j}(t) h_{j}\left\{\phi_{j k} n_{k}(t) h_{i j k}+\sum_{m=k+1}^{l} \phi_{j m} n_{m}(t) h_{m}\right\} .
\end{gathered}
$$

Here, $k$ is the unique integer satisfying

and

$$
v_{k-1 / 2}<v_{i+1 / 2}-v_{j}<v_{k+1 / 2},
$$

$$
h_{i j k}=v_{k+1 / 2}-\left(v_{i+1 / 2}-v_{j}\right) \text {. }
$$

$F\left(v_{i+1 / 2}, t\right)$ is now defined by Eqs. (14). We note that the formula (14c) "naturally" satisfies Eq. (14a), but not Eq. (14b). Making the special definition $F\left(v_{I+1 / 2}, t\right) \equiv 0$ establishes that no particles with mass greater than $v_{I+1 / 2}$ are created and has the following effect: if two particles coagulate to form a single particle whose volume is greater than $v_{l}$, then this mass is automatically placed into the proper number of particles in the $I-t h$ cell so that mass is conserved. Thus, in the absence of removal and sources, all mass will eventually settle into the $I-t h$ cell, as in the Nv method described in the previous section. 
In the remainder of this paper, we shall describe the method of Eqs. (13) and (14) as the " $\mathrm{Cr}$ " method - " $\mathrm{C}$ " referring to the fact that the method is based on the conservative form of the aerosol equation.

The approximation used to derive Eq. (14c) consists of taking $n$ to be constant on each cell. Physically, this can be interpreted as assuming that the aerosol particles are distributed uniformly within each cell, rather than all being located at the cell centers, as in the Nv method.

One can easily see that the number of mathematical operations required to evaluate the coagulation terms in Eq. (13) is significantly less than the number required to evaluate the corresponding terms in Eq. (8). This permits the $\mathrm{Cv}$ method to be solved more efficiently than the Nv method. We have observed this in our computer simulations, which we discuss in the following section.

\section{POSITIVITY}

One of the most important features of any successful numerical approximation is its ability to produce non-negative solutions. For our method to be reliable and stable, the number density obtained from solving Eq. (13) must be non-negative for all times. One way to ensure this is to examine carefully the behavior of the negative terms in Eq. (13) as $n_{i} \rightarrow 0$. A positive, stable method requires that these negative terms vanish as $n_{i}$ tends to zero. [This occurs with the Pardiseko method; see Eq. (8).]

If we examine Eq. (13), which can be written as

$$
\frac{d}{d t} v_{i} n_{i}(t) h_{i}=\Delta F_{i}(t)-v_{i} R_{i}(t) n_{i}(t) h_{i}+v_{i} S_{i}(t) h_{i} \quad,
$$

where

$$
\Delta F_{i}(t)=F\left(v_{i-1 / 2}, t\right)-F\left(v_{i+1 / 2}, t\right) \quad,
$$

we find that the removal term vanishes as $n_{i}$ tends to zero. Thus, for our method to be stable, $\Delta F_{i}(t)$ must be non-negative as $n_{i}$ tends to zero.

To show this, we rewrite Eq. (15b) using Eq. (14) as

$$
\begin{aligned}
\Delta F_{i}(t)= & \sum_{j=1}^{i-1} v_{j} n_{j}(t) h_{j}\left\{\phi_{j k^{\prime}} n_{k^{\prime}}(t) h_{i-1, j k^{\prime}}+\sum_{m=k^{\prime}+1}^{I} \phi_{j m} n_{m}(t) h_{m}\right\} \\
& -\sum_{j=1}^{i} v_{j} n_{j}(t) h_{j}\left\{\phi_{j k} n_{k}(t) h_{i j k}+\sum_{m=k+1}^{I} \phi_{j m} n_{m}(t) h_{m}\right\} .
\end{aligned}
$$

Here, $k$ and $k^{\prime}$ are the unique integers satisfying

Also,

$$
\begin{aligned}
& v_{k-1 / 2}<v_{i+1 / 2}-v_{j}<v_{k+1 / 2}, \\
& v_{k^{\prime}-1 / 2}<v_{i-1 / 2}-v_{j}<v_{k^{\prime}+1 / 2} .
\end{aligned}
$$

$$
h_{i j k}=v_{k+1 / 2}-\left(v_{i+1 / 2}-v_{j}\right) \text {, }
$$




$$
h_{i-1, j k^{\prime}}=v_{k^{\prime}+1 / 2}-\left(v_{i-1 / 2}-v_{j}\right)
$$

Eq. (15c) can be rearranged in the form

$$
\begin{aligned}
\Delta F_{i}(t) & =\sum_{j=1}^{i-1} v_{j} n_{j}(t) h_{j}\left\{\sum_{m=k^{\prime}}^{I} \phi_{j m} n_{m}(t) h_{m k^{\prime}}-\sum_{m=k}^{I} \phi_{j m} n_{m}(t) h_{m k}\right\} \\
& -v_{i} n_{i}(t) h_{i}\left\{\phi_{j k} n_{k}(t) h_{i j k}+\sum_{m=k+1}^{l} \phi_{j m} n_{m}(t) h_{m}\right\}
\end{aligned}
$$

where

and

$$
h_{m k^{\prime}}=\left\{\begin{array}{lr}
h_{m} & \text { if } m>k^{\prime} \\
h_{i-1, j k^{\prime}} & \text { if } m=k^{\prime}
\end{array}\right.
$$

$$
h_{m k}=\left\{\begin{array}{ll}
h_{m} & \text { if } m>k \\
h_{i j k} & \text { if } m=k
\end{array} .\right.
$$

The first term on the right side of Eq. (16a) represents the rate at which aerosol particles are produced in $\Delta v_{i}$ about $v_{i}$ due to the coagulation of other particles, whereas the second term represents the rate at which particles in $\Delta v_{i}$ about $v_{i}$ are removed due to coagulation with other particles.

Since $k$ is always greater than or equal to $k^{\prime}$, then the first term on the right side of Eq. (16a) is always non-negative. Also, as $n_{i}$ tends to zero, the second term on the right side of Eq. (16a) vanishes. This establishes that $\Delta F_{i}(t)$ is non-negative as $n_{i}$ tends to zero, as required.

\section{NUMERICAL RESULTS}

In the numerical calculations involving each of the methods developed above, we used a standard fourth-order Runge-Kutta method (Anderson (1974)) to discretize $t$ in conjunction with the $\mathrm{Nv}$ and $\mathrm{Cv}$ methods for discretizing $v$.

We shall now describe numerical results for a problem in which the exact analytic particle number density is known for all times. This solution exists if the coagulation kemel is independent of the aerosol volume, the source and removal rates are equal to zero, and the initial condition is a simple exponential function:

$$
\begin{gathered}
\phi(u, v)=\phi_{0}, \\
S(v, t)=0, \\
R(v, t)=0, \\
n_{0}(v)=\frac{N_{0}}{v_{0}} \exp \left(-\frac{v}{v_{0}}\right) .
\end{gathered}
$$

Here $N_{0}$ is the prescribed total number of particles and $v_{0}$ is the prescribed average particle volume at $t=0$. The exact solution of Eq. (1) with these definitions is Williams (1990)) 


$$
n(v, t)=\frac{N(t)}{u(t)} \exp \left(-\frac{v}{u(t)}\right)
$$

where

$$
N(t)=\int_{0}^{\infty} n(v, t) d v=\frac{N_{0}}{1+\frac{\phi_{0} N_{0}}{2} t},
$$

and

$$
u(t)=\frac{\int_{0}^{\infty} v n(v, t) d v}{\int_{0}^{\infty} n(v, t) d v}=\frac{N_{0} v_{0}}{N(t)}
$$

In our numerical calculations, we used the values (Williams (1986)) $v_{0}=1,6464 \times 10^{-19}$ $\mathrm{c} \mathrm{m}^{3}, N_{0}=9.15 \times 10^{13} \mathrm{~m}^{-3}$, and $\phi_{0}=k T / 3 \eta$, where $k=1.38 \times 10^{-23} \mathrm{~J} \mathrm{~K} \mathrm{~K}^{-1}$ is Boltzmann's constant $T=$ temperature $=300^{\circ} \mathrm{K}$, and $\eta=$ viscosity of the medium $=$ $1.8 \times 10^{-5} \mathrm{~kg} \mathrm{~m}^{-1} \mathrm{sec}^{-1}$. We also used $v_{\min }=10^{-24} \mathrm{~cm}^{3}$.

This exact solution, initially and after one, five, and ten hours, is plotted in Fig. 1.

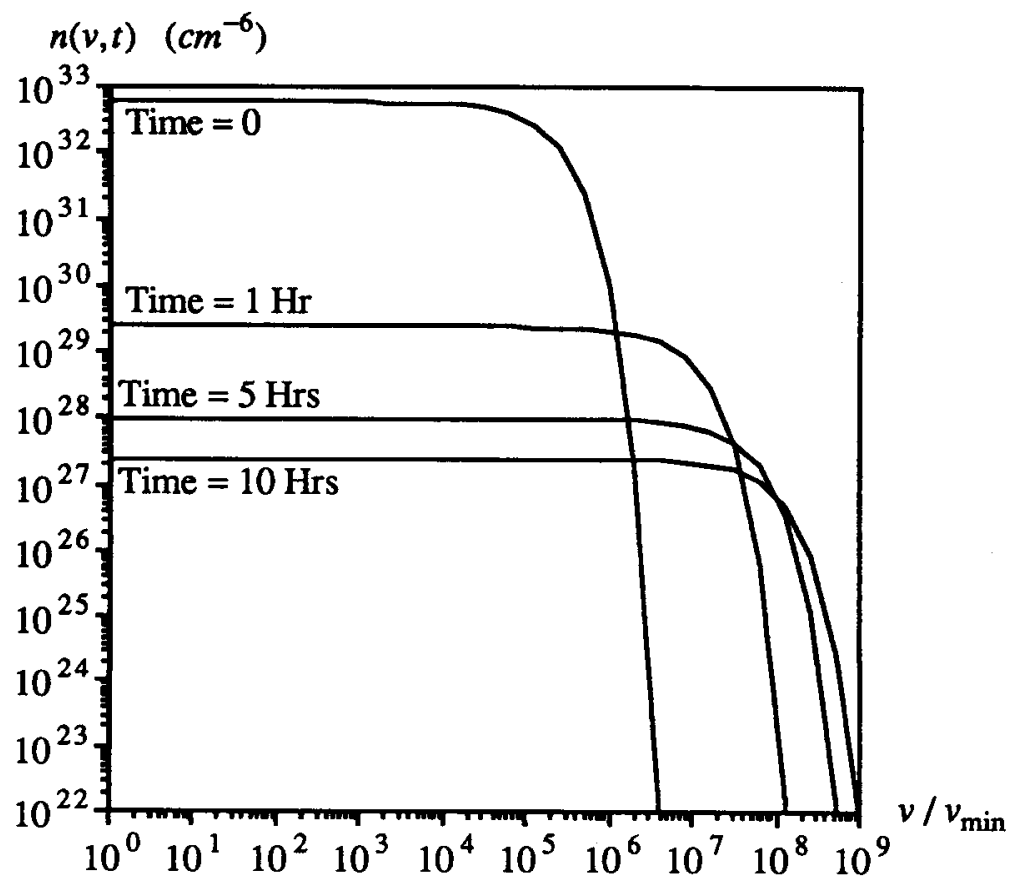

Figure 1: Exact $n(v, t)$ 
This shows that as a result of the coagulation process, more large particles are created that subsequently are involved in the coagulation process. Therefore, to accurately simulate the problem for sufficiently large times, one must use a sufficiently large value of $v_{\max }$. For this special problem, we see that if the duration of interest is ten hours, then we must consider a volume range that spans nine order of magnitude. We used this range in our numerical calculations. Thus, with $\nu_{\min }=10^{-24} \mathrm{~cm}^{3}$, we have $v_{\max }=10^{-15} \mathrm{~cm}^{3}$.

Since the total mass in this problem is conserved (there are no source and removal terms), the system evolves in time from one having a large number of small particles to one having a smaller number of larger particles. The relative total number of particles in the system as a function of time is displayed in Fig. 2.

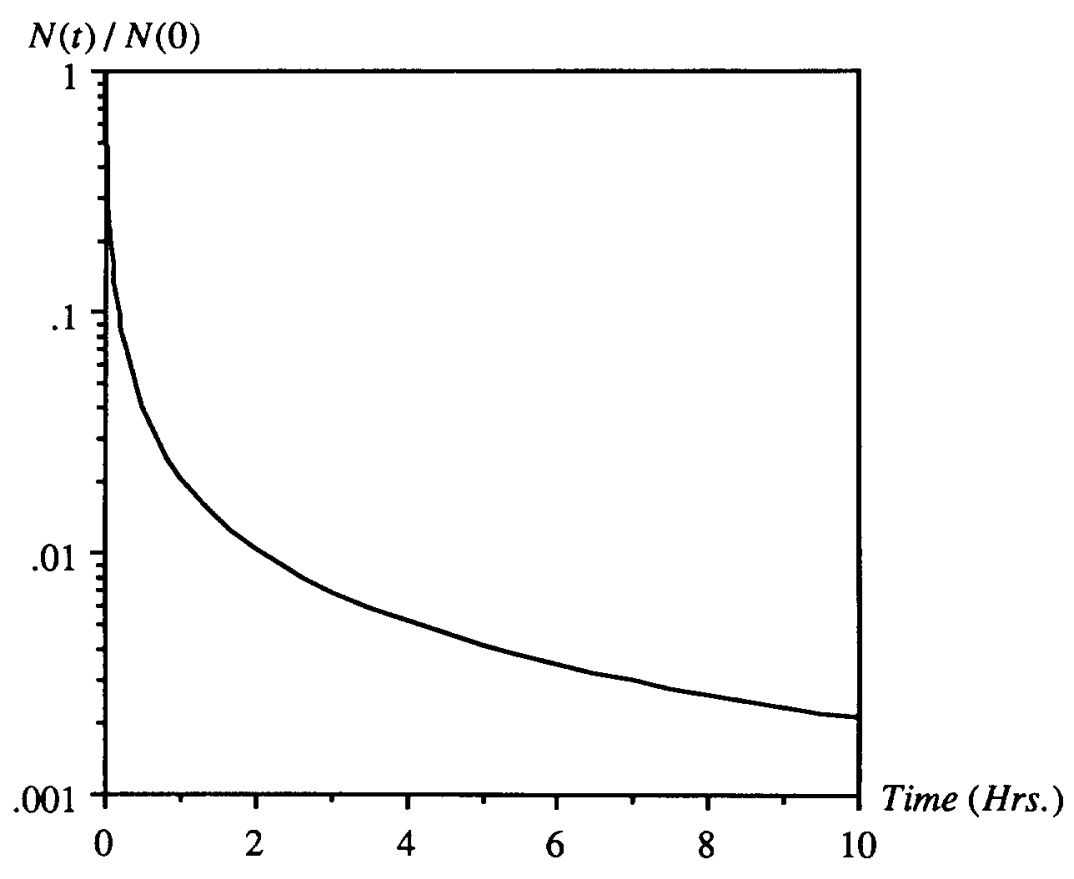

Figure 2: Exact $N(t) / N(0)$

We ran the problem described above, using the logarithmic grid defined by Eqs. (4) with $\mathrm{I}=50$ cells. If we define

$$
\begin{aligned}
& \text { Relative error of the total particle density }=\left|\frac{N_{\text {exact }}(t)-N(t)}{N_{\text {exact }}(t)}\right|, \\
& N(t)=\sum_{i=1}^{I} n_{i}(t) h_{i},
\end{aligned}
$$

then the results shown in Fig. 3 illustrate that the $\mathrm{Cv}$ method produces a more accurate total particle density than the Nv method. 


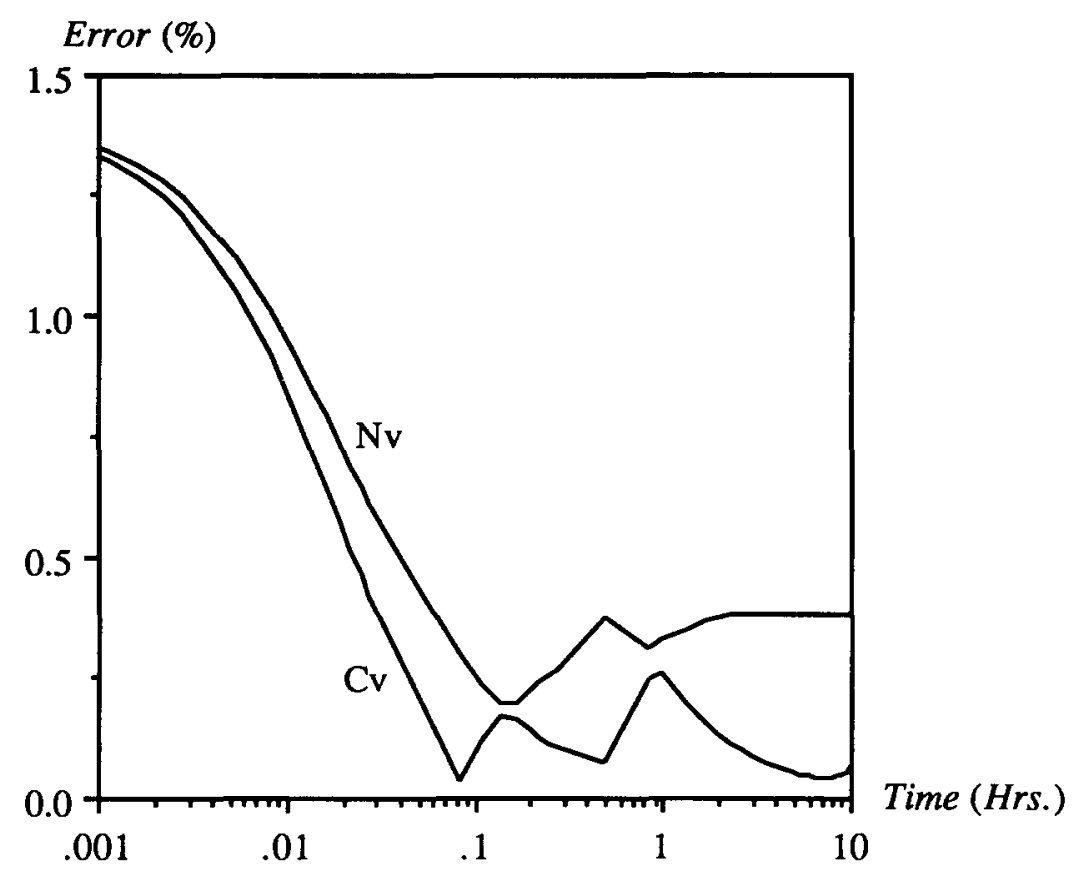

Figure 3: Relative Error in $N(t)$

If we define:

$$
\text { Relative error in the particle spectrum }=\left|\frac{n_{\text {exact }}(v, t)-n(v, t)}{n_{\text {exact }}(v, t)}\right|,
$$

then Figs. 4 - 6 display these relative errors for the Nv and $\mathrm{Cv}$ methods for $t=1,5$, and 10 hours. We see that the the Cv method generally produces a more accurate particle spectrum than the Nv method, particularly for small $v$.

Finally, in Table 1, we present timing results obtained by solving the above problem on three grids $(25,50$, and 100 cells), for $t=10$ hours, on an Apollo DN4000 computer. These results show that the $\mathrm{Cv}$ method is about three times faster than the Nv method. This is due to the simpler algebraic structure of the Cv method, as discussed in the previous section.

\section{CONCLUSIONS}

In this paper we have developed a new approach to the numerical simulation of nuclear aerosol problems by using a "conservative" form of the kinetic aerosol equation. Although the conservative and original forms of the aerosol equation are mathematically equivalent, they generate numerical approximations that are not mathematically equivalent. We have shown that numerical methods obtained from the conservative form of the equation have advantages over methods obtained from the original form of the equation. A particular advantage of the conservative form is that numerical methods can easily (without 


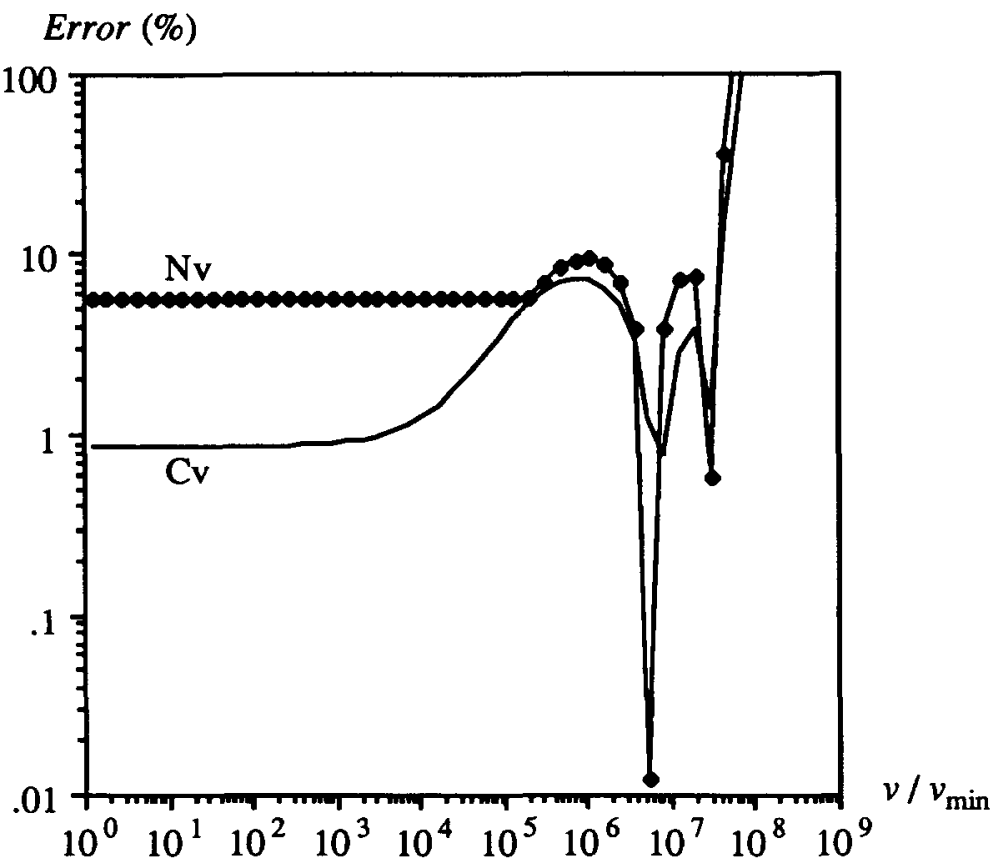

Figure 4: Relative Error in Particle Spectrum for $t=1 \mathrm{Hr}$.

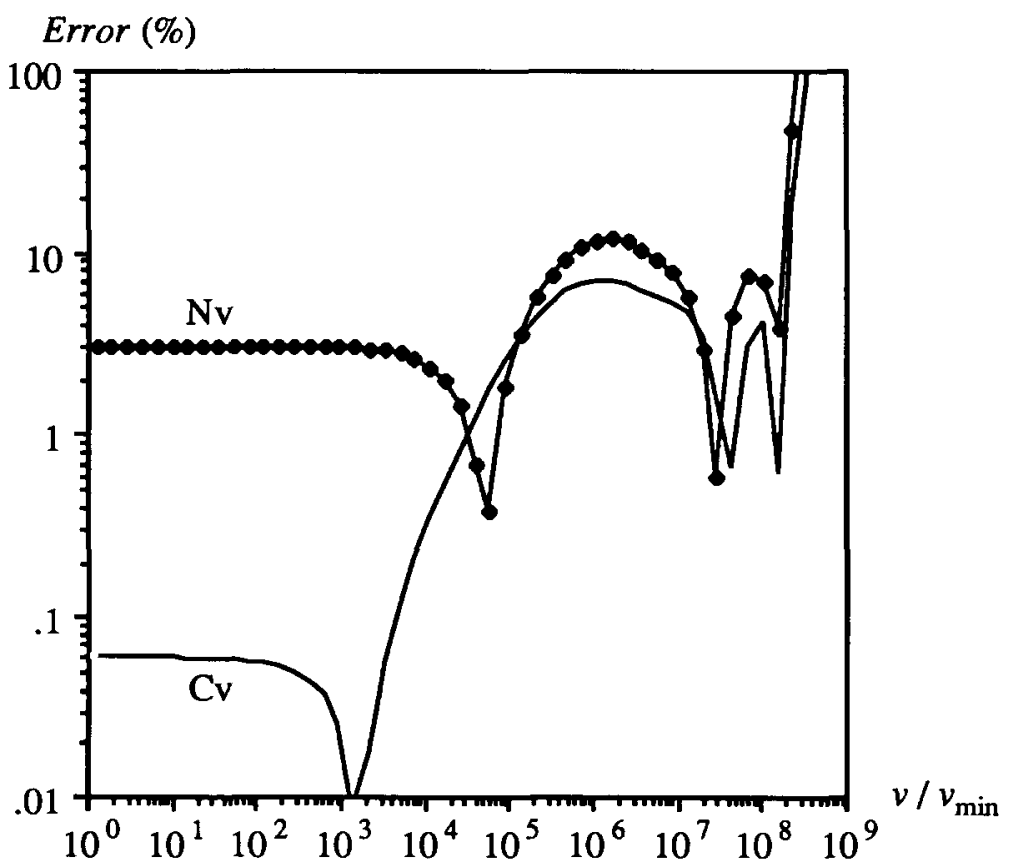

Figure 5: Relative Error in Particle Spectrum for $t=5 \mathrm{Hrs}$. 


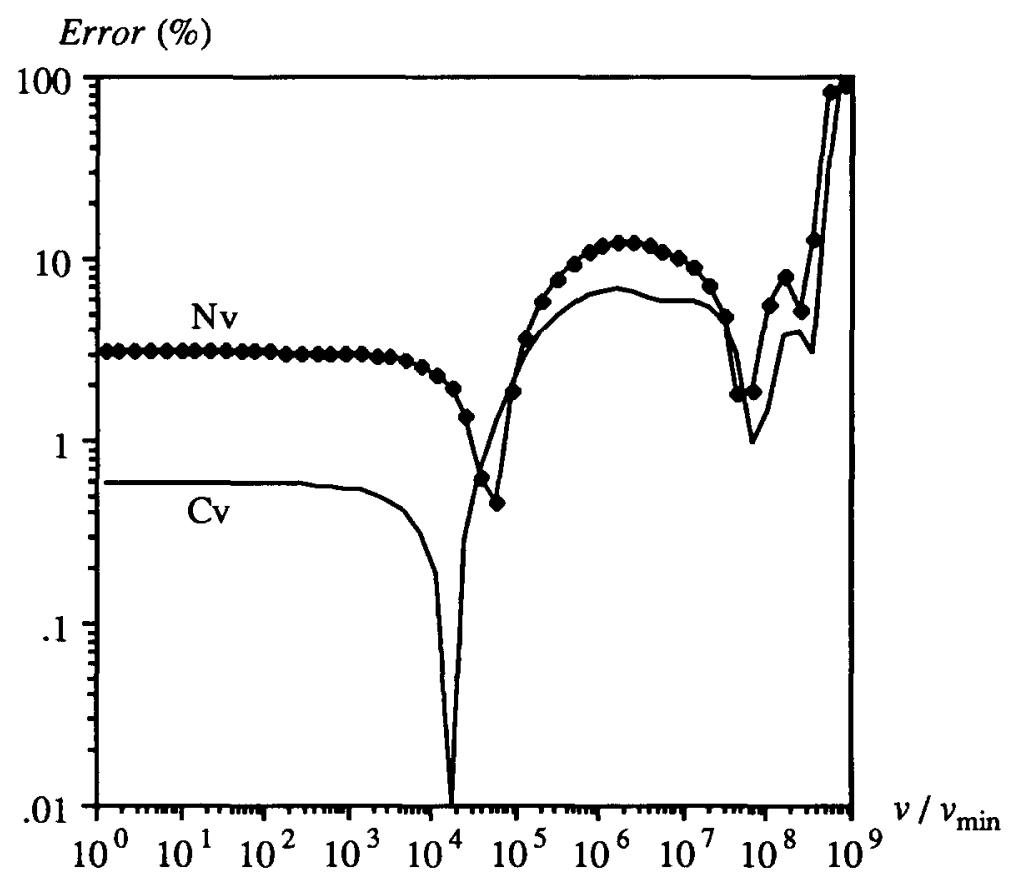

Figure 6: Relative Error in Particle Spectrum for $t=10 \mathrm{Hrs}$.

\begin{tabular}{|c|c|c|}
\hline \multirow{2}{*}{$\begin{array}{l}\text { Number } \\
\text { of Cells }\end{array}$} & \multicolumn{2}{|c|}{ Method } \\
\cline { 2 - 3 } & $\mathrm{Nv}$ & $\mathrm{Cv}$ \\
\hline 25 & 11 & 5 \\
50 & 45 & 14 \\
100 & 223 & 55 \\
\hline
\end{tabular}

Table 1: Timing Comparison (in sec) of the $\mathrm{Nv}$ and $\mathrm{Cv}$ Methods.

making any unnecessary physical assumptions) be derived for it that conserve mass. We have shown that a simple such method is both more accurate and more efficient than a corresponding method based on the nonconservative form of the kinetic aerosol equation.

More sophisticated numerical methods can certainly be developed for the conservative form of the kinetic aerosol equation. For example, linear finite element methods have been developed for the nonconservative form of the equation (Dunbar 
(1984)), and there is no doubt that these can be systematically developed for the conservative form of the equation. However, we will not pursue this here.

In summary, the conservative form of the kinetic aerosol equation is useful for generating accurate and efficient numerical schemes. We have observed no apparent disadvantages to numerical methods based on this form of the equation.

\section{ACKNOWLEDGEMENTS}

We would like to thank Dr. M.M.R.Williams for his help in the preliminary stages of this research. Also, we would like to acknowledge the comments of a referee, which led to the results in Sec. V. Work by the first author (K.N.A) was supported by the Ministry of Higher Education and Scientific Research, Baghdad, Iraq. Work by the second author (E.W.L.) was supported by the National Science Foundation Grant EET-8721680.

\section{REFERENCES}

1. Silberberg M., editor (1979) "Nuclear Aerosols in Reactor Safety", A State of Art Report by a Group of Experts of the NEA Committee on the Safety of Nuclear Installations, Nuclear Energy Agency, Organization for Economic Co-operation and Development (OECD) .

2. Abbey F., chairman (1985) "Nuclear Aerosols in Reactor Safety", Supplement to the June 1979 Report by a Group of Experts of the NEA Committee on the Safety of Nuclear Installations, Nuclear Energy Agency, Organization for Economic Cooperation and Development (OECD).

3. Dunbar I. H. and Fermandjian J. (1984) "Comparison of Sodium Aerosol Codes", EUR 9172 EN.

4 Bunz H. (1984) "PARDISEKO IV - A Computer Code for Calculating the Aerosol Behavior in Closed Vessels", KfK 3545e.

5. Williams M.M.R. (1966) The Slowing Down and Thermalization of Neutrons, North-Holland, Amsterdam.

6. Anderson N., Bjorck A., and Dahlquist G. (1974) Numerical Methods, Prentice Hall, Inc., Englewood Cliffs, N.J.

7. Williams M.M.R. and Loyolka S.K. (1990) Aerosol Science, Pergamon Press, Oxford.

8. Williams M.M.R. (1986) Prog. Nucl. Energy, 17, 1. 